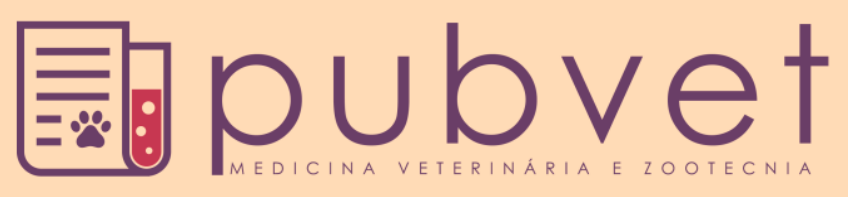

https://doi.org/10.22256/pubvet.v12n2a36.1-4

\title{
Megabacteria (Macrorhabdus ornithogaster) em psitacídeos mantidos em estabelecimentos comerciais do município de Uberaba - MG
}

\author{
Isabella Hercos de Paula ${ }^{0} *$, Flávia Prata Linhares $\bullet 1$, Cláudio Yudi Kanayama ${ }^{\bullet}$, \\ Sophia Prata de Carvalho ${ }^{1}$, Eustáquio Resende Bittar ${ }^{\bullet}$, Marco Túlio Gomes \\ Campos $\bullet 1$, Joely Fereira Figueredo Bittar $\bullet 3$ \\ ${ }^{1}$ Médico(a) Veterinário (a), Universidade de Uberaba, MG. E-mail: flaviaprata13@hotmail.com, s.prata@hotmail.com, \\ camposmtg@gmail.com \\ ${ }^{2}$ Professor, Mestre Curso Medicina Veterinária Universidade de Uberaba, MG. E-mail: claudio.kanayama@uniube.br \\ ${ }^{3}$ Professor, Doutor Curso Medicina Veterinária e do Programa de Mestrado em Sanidade e Produção Animal nos Trópicos da Universidade \\ de Uberaba, MG.E-mail: joely.bittar@uniube.br,eustaquio.bittar@uniube.br \\ *Autor para correspondência, E-mail: isabellahp@msn.com
}

\begin{abstract}
RESUMO. A megabacteriose é uma doença que acomete algumas espécies de aves, tendo como agente etiológico Macrorhabdus ornithogaster, um microrganismo Gram positivo de forma bacilar e com características de fungo, sendo classificado como fungo ascomiceto anamórfico. É um agente oportunista que se torna patogênico em casos de imunossupressão, manifestando assim a sua forma clínica, que varia muito de acordo com cada animal, podendo levar o animal ao óbito. Neste contexto, o presente trabalho objetivou avaliar a prevalência de megabacteria em psitacídeos criados em estabelecimentos comerciais do município de Uberaba-MG associado as condições de criação. Para isso, foram colhidas amostras de secreções do ventrículo e pro ventrículo de 71 aves, entre elas Calopsitas (Nymphicus hollandicus) ( $\mathrm{n}=33$ ), Periquitos Australianos (Melopsittacus undulatus) $\quad(\mathrm{n}=31)$, Agapornis (Agapornis roseicollis) $(\mathrm{n}=4)$ e Catarinas (Bolborhynchuslineola) $(\mathrm{n}=3)$ e transferidas para lâminas de vidro. As amostras foram encaminhadas para o Laboratório de Medicina Veterinária Preventiva do Hospital Veterinário de Uberaba, para pesquisa do patógeno. Após coloração das lâminas por Gram pode-se notar a presença do microrganismo em 35,21\% das aves analisadas. A prevalência entre as Calopsitas, Periquitos Australianos, Agapornis e Catarinas foi respectivamente de: 42,42\% (14/33), 29,03\% (9/31), 50\% (2/4) e 0\% (0/3). Neste contexto, pode-se concluir que a Macrorhabdus ornithogaster é comum entre as aves, principalmente entre as Calopsitas e o manejo incorreto dos animais pode ser a causa da elevada prevalência. Nos estabelecimentos comerciais observaram-se as condições das gaiolas, qualidade dos bebedouros e comedouros, quantidade de fezes na gaiola e número de animais por gaiola inadequados.
\end{abstract}

Palavras chave: Diagnóstico, fungo, megabacteriose

\section{Megabacteria (Macrorhabdus ornithogaster) in psittacids kept in commercial establishments in the municipality of Uberaba - MG}

\begin{abstract}
Megabacteriosis is a disease that affects some species of birds, having as etiological agent Macrorhabdus ornithogaster, a gram positive microorganism of bacillary form and with characteristics of fungus, being classified as an anamorphic asymmetric fungus. It is an opportunistic agent that becomes pathogenic in cases of immunosuppression, thus manifesting its clinical form, which varies greatly according to each animal, which can lead to death. In this context, the present study aimed to evaluate
\end{abstract}


the prevalence of megabacteria in psittacids kept in commercial establishments in the municipality of Uberaba, state of Minas Gerais, associated with the conditions of breeding. For this, samples of secretions of the ventricle and proventriculus of 71 birds were collected, among them Nymphicus hollandicus $(\mathrm{n}=33)$, Melopsittacus undulatus $(\mathrm{n}=31)$, Agapornis roseicollis $(\mathrm{n}=4)$ Bolborhynchuslineola $(\mathrm{n}=3)$ and transferred to glass slides. The samples were sent to the Laboratory of Preventive Veterinary Medicine of the Veterinary Hospital of Uberaba, to investigate the pathogen. After staining of the slides by Gram, it can be noticed the presence of the microorganism in $35.2 \%$ of the birds analyzed. The prevalence among the Cockatiels, Budgerigars, Lovebirds and Barred parakeet were respectively: $42.4 \%$ (14/19), 29.0\% (9/22), 50.0\% (2/4) and 0\% (0/3). In this context, it can be concluded that Macrorhabdus ornithogaster is common among birds, especially among cockatiels and the incorrect handling of the animals may be the cause of the high prevalence. Commercial establishments were observed as conditions of cages, quality of drinking fountains and feeders, amount of feces in the cage and number of animals per inadequate cage.

Key words: Diagnosis, fungi, megabacteriosis

\section{Megabacteria (Macrorhabdus ornithogaster) en psitácidos mantenidos en establecimientos comerciales del municipio de Uberaba - MG}

RESUMEN. La megabacteriosis es una enfermedad que afecta a algunas especies de aves, teniendo como agente etiológico Macrorhabdus ornithogaster, un microorganismo Gram positivo de forma bacilar y con características de hongo, y se clasifica como hongo ascomiceto anamórfico. Es un agente oportunista que se vuelve patogénico en casos de inmunosupresión, manifestando así su forma clínica, que varía mucho de acuerdo con cada animal, pudiendo llevarlo al óbito. En este contexto, el presente trabajo tuvo como objetivo evaluar la prevalencia de megabacteria en psitácidos criados en establecimientos comerciales del municipio de Uberaba estado de Minas Gerais asociado a las condiciones de producción. Para ello, se tomaron muestras de secreciones del ventrículo y proventrículo de 71 aves, entre ellas Nymphicus hollandicus $(\mathrm{n}=33)$, Melopsittacus undulatus $(\mathrm{n}=31)$, Agaporni sroseicollis $(\mathrm{n}=4)$ y Bolborhynchus lineola $(\mathrm{n}=3)$ y transferidas para láminas de vidrio. Las muestras fueron encaminadas al Laboratorio de Medicina Veterinaria Preventiva del Hospital Veterinario de Uberaba, para investigación del patógeno. Después de la coloración de las láminas por Gram se puede notar la presencia del microorganismo en el $35,21 \%$ de las aves analizadas. La prevalencia entre las Cacatúas ninfas, Periquitos común Agapornis, Pericos barreteados fue, respectivamente, del 42,4\% (14/33), 29,0\% (9/31), $50.0 \%$ (2/4) y 0\% (0/3). Se puede concluir que el Macrorhabdus ornithogaster es común entre las aves, principalmente entre las Cacatúas ninfas y el manejo incorrecto de los animales puede ser la causa de la elevada prevalencia. Los establecimientos comerciales se observaron condiciones de las jaulas, calidad de los bebederos y comederos, cantidad de heces en la jaula y número de animales por jaula inadecuados.

Palabras clave: Diagnóstico, hongo, megabacteriosis

\section{Introdução}

A megabacteriose, também conhecida como "Síndrome Light Going" tem como agente etiológico Macrorhabdus ornithogaster, um microrganismo Gram positivo em forma de bacilo, porém de tamanho maior (Queirós et al., 2013). De acordo com suas características, foi classificado como um fungo ascomiceto anamórfico, que habita principalmente o proventrículo e ventrículo de diversas aves, mas que também pode ser encontrado em glândulas superficiais (uropigiais e anexas) das aves (Cubas et al., 2014). É uma doença oportunista de distribuição mundial em aves, tanto doméstica quanto selvagens (Goossens, 2015). Sua sintomatologia depende do sistema imunológico de cada ave, sendo algumas assintomáticas e outras podendo apresentar sinais 
clínicos, tais como, emagrecimento progressivo, regurgitação, mudança no aspecto das fezes, apatia e pequenas hemorragias levando ao óbito (Queirós et al., 2013).

Para diagnosticar clinicamente a megabacteriose, devem-se olhar atentamente as fezes, se estiverem com aspecto amolecido ou de coloração anormal é indício da presença da doença. Outros fatores clínicos importantes como diagnóstico são as penas arrepiadas, aves apáticas, tristes e o papo vazio mesmo após terem se alimentado. O diagnóstico definitivo é feito com o isolamento do microrganismo, ou pelo esfregaço das fezes ou do pro ventrículo com a coloração de Gram ou PAS (Cubas et al., 2014, Balani \& Cubas, 2016).

Este trabalho foi para avaliar a prevalência de Macrorhabdus ornithogaster em aves criadas em cativeiros da região de Uberaba-MG e correlacionar com as condições de criação.

\section{Material e Métodos}

O trabalho foi realizado em 71 psitacídeos criados em estabelecimentos comerciais na cidade de Uberaba. Sendo 33 Calopsitas (Nymphicus hollandicus), 31 Periquitos Australianos (Melopsittacus undulatus), 4 Agapornis (Agapornis roseicollis) e 3 Catarinas (Bolborhynchuslineola).

Antes da coleta das amostras, os animais foram avaliados em relação à alteração comportamental, local em que habitavam presença dos coabitantes e condições sanitárias do ambiente. Os animais foram capturados aleatoriamente e contidos corretamente, pelas patas e cabeça na região parietal para que não machuque o animal e o veterinário, não comprimindo o tórax, deixando assim livre para sua respiração. Pois as aves não apresentam diafragma e seus movimentos respiratórios são basicamente realizados pela musculatura peitoral.

Amostras de secreções foram coletadas da parede do ventrículo e proventrículo das aves, com swab estéril.

O material foi transferido para uma lâmina de vidro e encaminhado ao Laboratório de Medicina Veterinária Preventiva do Hospital Veterinário de Uberaba. As lâminas contendo as secreções do ventrículo e proventrículo foram submetidas à coloração de Gram

Nos criatórios observaram-se as condições das gaiolas, qualidade dos bebedouros e comedouros, quantidade de fezes na gaiola e poleiro e número de animais por gaiola inadequados.

\section{Resultados}

Das 71 aves avaliadas, $25(35,2 \%)$ eram positivas para megabacteria e $46(64,8 \%)$ eram negativas. Em relação às espécies de aves avaliadas a prevalência de megabacteria em Calopsitas (Nymphicus hollandicus) foi de $42,4 \%$ (14/33), em Periquitos australianos (Melopsittacus undulatus) de 29,0\% (9/31), em Agapornis (Agapornis roseicollis) 50,0\% (2/4) e Catarinas (Bolborhynchus lineola) 0\% (0/3).

Em relação às gaiolas, bebedouros e comedouros observaram-se precárias condições sanitárias em todos os criatórios analisados, apresentou-se excesso de fezes nas gaiolas, poleiros e número elevado de animais por gaiola, visualizado pelo bater dos animais um nos outros durante o voo.

\section{Discussão}

A prevalência observada no presente estudo foi menor que a observada em outros estudos. Um estudo realizado em 2013 observou 48\% de aves positivas (Queirós et al., 2013), e outro realizado em 2000, 56,3\% das aves apresentaram megabacteria (Werther et al., 2000). Vários autores colhem materiais das fezes, pois acreditam que a megabacteria além de ter seu habitat no proventrículo e ventrículo, tenha predileção também nas fezes (Carvalho et al., 2011). Nos estudos realizados em 2013 de 39 aves apenas duas $(5,1 \%)$ foram positivas para megabacteria no exame de fezes e eram assintomáticos, Conclui-se que a pesquisa de Macrorhabdu sornithogaster em secreções do proventrículo e ventrículo tem maior sensibilidade (Lanzarot et al., 2013).

Apesar do elevado percentual de positividade em Calopsitas (Nymphicus hollandicus), nenhuma apresentou sinais clínicos aparentes, assim como foi citado em alguns estudos, que muitas aves aparentemente saudáveis costumam ser portadoras de megabacteria (Lanzarot et al., 2013, Queirós et al., 2013).

O aumento da propagação da doença se dá pelo manejo incorreto, como por exemplo: aves de espécies diferentes, aglomeradas e com condições sanitárias ruins, que podem ser um fator agravante para sua transmissão (Queirós et al., 2013). Tais fatos corroboram com os resultados do presente trabalho, em que ocorreu uma maior porcentagem de animais positivos naqueles que eram mantidos 
aglomerados, enquanto que em locais onde havia menor aglomeração de animais (um a dois em cada gaiola), o resultado positivo para megabacteria foi menor.

\section{Conclusões}

Com base nos resultados obtidos pode-se concluir que:

Aves mantidas em estabelecimentos comerciais de Uberaba apresentam megabacteria (Macrorhabdus ornithogaster).

Calopsitas (Nymphicus hollandicus) apresentam maior prevalência por Macrorhabdus ornithogaster que Periquitos Australianos (Melopsittacus undulatus) e Agapornis (Agapornis roseicollis).

Catarinas (Bolborhynchus lineola) não apresentam Macrorhabdus ornithogaster devido à baixa aglomeração.

\section{Referências Bibliográficas}

Balani, V. \& Cubas, Z. S. 2016. Megabactéria e os prejuízos que ela pode causar no plantel. Revista Aves e Criadores, 24, 48-49.

Carvalho, P. R., Queirós, T. S. \& Pita, M. C. G. 2011. Megabacteriose em aves. Pesquisa \& Tecnologia, 8, 1-2.

Cubas, Z. S., Silva, J. C. R. \& Dias, J. L. C. 2014. Tratado de animais selvagens-medicina veterinária. Editora Roca.
Goossens, C. 2015. Le point sur macrorhabdus ornithogaster, agent étiologique de la mégabactériose aviaire. Médecine vétérinaire. Toulouse - ENVT, Ecole Nationale Vétérinaire de Toulouse.

Lanzarot, P., Blanco, J. L., Alvarez-Perez, S., Abad, C., Cutuli, M. T. \& Garcia, M. E. 2013. Prolonged fecal shedding of 'megabacteria'(Macrorhabdus ornithogaster) by clinically healthy canaries (Serinus canaria). Medical Mycology, 51, 888-891.

Queirós, T. S., Carvalho, P. R. \& Pita, M. C. G. 2013. Megabacteriose: Macrorhabdus ornithogaster em aves - Revisão. PUBVET, 7, $1-10$.

Werther, K., Schocken-Iturrino, R. P., Verona, C. E. S. \& Barros, L. S. S. 2000. Megabacteriosis occurrence in budgerigars, canaries and lovebirds in Ribeirao Preto region-Sao Paulo state-Brazil. Revista Brasileira de Ciência Avícola, 2, 183-187.

Article History:

Received 03 October 2017

Accepted 17 November 2017

Available online 25 January 2018

License information: This is an open-access article distributed under the terms of the Creative Commons Attribution License 4.0, which permits unrestricted use, distribution, and reproduction in any medium, provided the original work is properly cited. 